Working Paper 09-20

Economic Series ( 12 )

February 2009
Departamento de Economía

Universidad Carlos III de Madrid

Calle Madrid, 126

28903 Getafe (Spain)

Fax (34) 916249875

\title{
Resistance to learning and the evolution of Cooperation
}

\author{
Raúl Jiménez ${ }^{\star}$ Haydée Lugo ${ }^{\dagger}$ Maxi San Miguel $^{\ddagger}$
}

\begin{abstract}
In many evolutionary algorithms, crossover is the main operator used in generating new individuals from old ones. However, the usual mechanism for generating offsprings in spatially structured evolutionary games has to date been clonation. Here we study the effect of incorporating crossover on these models. Our framework is the spatial Continuous Prisoner's Dilemma. For this evolutionary game, it has been reported that occasional errors (mutations) in the clonal process can explain the emergence of cooperation from a non-cooperative initial state. First, we show that this only occurs for particular regimes of low costs of cooperation. Then, we display how crossover gets greater the range of scenarios where cooperative mutants can invade selfish populations. In a social context, where crossover involves a general rule of gradual learning, our results show that the less that is learnt in a single step, the larger the degree of global cooperation finally attained. In general, the effect of step-by-step learning can be more efficient for the evolution of cooperation than a full blast one.
\end{abstract}

keywords: Evolutionary games, Continuous Prisoner's Dilemma, Spatially structured populations, Crossover, Learning.

* Departamento de Estadística, Universidad Carlos III de Madrid, Getafe, Spain, email: raul.jimenez@uc3m.es

Departamento de Economía, Universidad Carlos III de Madrid, Getafe, Spain, email: hlugo@eco.uc3m.es

* IFISC (CSIC-UIB), Campus Universitat Illes Balears, Palma de Mallorca, Spain, email:maxi@i_sc.uib-csic.es 


\section{Introduction}

The evolution of cooperation has been a challenging problem since Darwin (Trivers, 1971; Axelrod \& Hamilton, 1981; Maynard \& Szathmáry, 1995; Dugatkin, 1997; Nowak, 2006). With sophisticated tools of evolutionary game theory, researchers from biology to behavioral sciences have attempted to shed light on the underlying mechanisms that outperform the vulnerability of cooperation of being cheated (Pennisi, 2005). Models with spatial structures have been considered by these researches to mimic real population where individuals do not interact with everybody else (Lieberman et al., 2005). In these models, individuals are located on the nodes of a network, play repeatedly with their neighbors, and update their strategies by copying (with occasional errors) the strategy of some more successful neighbor. Different strategy update rules respond to the same evolutionary principle of clonal reproduction of successful phenotypes (Szabó \& Fáth, 2007) and are usually implemented through two basic operators: Selection, in which individuals identify whom to copy, and mutation, occasional errors in the copy process.

Discrete-choice models, where cooperation is all or nothing, do not lead mutation to generating new phenotypes into the population. However in more realistic models, in which individuals can exhibit variable degrees of cooperation, mutation can be certainly a source of generating diversity. The study of cooperative behavior in a quantitative way rather than a qualitative way has been crucial in dealing with the problem of evolution and the stability of cooperation. The first studies on this issue (Verhoeff, 1993; Mar \& St Denis, 1994; Frean, 1996) considered the evolution of degrees of cooperation by interpolating payoffs between the discrete outcomes of the classical Prisoner's Dilemma model (PD). Later, a natural approach to model variable levels of cooperation was introduced by Doebeli \& Knowlton (1998) and Roberts \& Sherratt (1998). Iteration and spatial structures based on continuous cooperative investment have also been addressed by Killingback et al. (1999); Wahl \& Nowak (1999a,b); Sherratt \& Roberts (1999); Koella (2000); Killingback \& Doebeli (2002); Le Gaillard et al. (2003); Ifti et al. (2004). Sherratt \& Roberts (2002) and Doebeli \& Hauert (2005) provide a complete review of previous researches on the stability in cooperation in variable-investment systems. In the iterated models, it is still necessary to have individuals capable of designing sophisticated strategies to play repeatedly using the past history. Such assumptions can be implausible in an evolutionary scenario. Developing some of the ideas of Doebeli \& Knowlton (1998), and following the classical scheme of Nowak \& May (1992) for 
spatial evolutionary games, Killingback et al. (1999) introduced the Continuous Prisioner's Dilemma (CPD). The joint consideration of continuous cooperative investments and spatial structured populations in their model represents an important advance in explaining the evolutionary origin of cooperation.

The strategy update rule considered in the seminal work of Nowak \& May (1992) was originally designed for the discrete PD, where clonation is the only way to generate offsprings. However, clonal reproduction is relatively rare among higher organisms, where crossover is the form of reproduction. Here we investigate the effect of introducing crossover in the strategy update rule of the spatial CPD. In particular, we use arithmetic crossover, the most elemental and universal crossover operator when phenotypes are represented by real numbers (Eiben \& Smith, 2007), such as investments considered in the CPD. Thus, the investment of an offspring is generated by merging the investments of her parents. In a social and economic context, where the copying (or imitation) is interpreted as learning (Ifti et al., 2004), arithmetic crossover can be viewed as a process of gradual learning. It seems natural that individuals hesitate to unconditionally imitate the investment of a neighbor. In adverse conditions in which costs for cooperation are high, individuals have reason to avoid the blind imitation of higher-investing partners, despite how successful they can be. Counterintutively, we show that this wariness leads gradual learning to being more efficient than a full blast imitation precisely in regimes of non-low costs of cooperation. For our analysis we require a deep review of the CPD. The paper is organized as follows: In section 2, we introduce the basic model, as well as general assumptions and the notation used throughout the paper. In section 3 , we characterize the different payoff regimes according to the asymptotic behavior of the CPD. In particular, we prove that previous results in which it is reported that cooperation gradually increases from a non-cooperative state correspond to particular payoff regimes that we classify as regimes of low cost. In section 4, we introduce crossover in the original model and explain how this mechanism operates for the maintenance and promotion of cooperation. We also provide the conditions in which higher-investment mutants can invade a population of selfish individuals. Section 5 summaries our conclusions. 


\section{The CPD in spatially structured populations}

In the CPD, if an individual, who invests $x$, interacts with another, who invests $y$, the former receives a payoff equal to $S(x, y)=B(y)-C(x)$ and the latter $S(y, x)=B(x)-C(y)$. The function $B(\cdot)$ specifies the benefit that an individual obtains from the investment made by the other in their pairwise interaction, and the function $C(\cdot)$ specifies the cost incurred by her. As standard assumptions, functions $B(\cdot)$ and $C(\cdot)$ are increasing and concave, with $B(0)=C(0)=0$, and $B(x) \geq C(x)$ for any feasible investment $x$ (Doebeli \& Hauert, 2005). In order to determine the maximal possible investment, it is required to compute the optimal mutual investment $x_{\max }$ that maximizes $B(x)-C(x)$. In general, for all $0<x<y<x_{\max }$, one can verify the inequalities shown by Killingback et al. (1999)

$$
S(y, x)<S(x, x)<S(y, y)<S(x, y),
$$

which are continuous versions of the well known conditions for the payoffs of the classical Prisoner's Dilemma (Killingback \& Doebeli, 2002).

To avoid the problem of comparing investments in different scales, we only consider investments in $[0,1]$. For that, it is sufficient to have $x_{\max } \geq 1$, therefore (1) holds for any pair of investments $0<x<y<1$.

Although the main results of this work cover general increasing-concave functions, the simulations shown throughout this paper are based on linear costs, namely, $C(x)=C x$, and benefit functions of the form $B(x)=$ $a[1-\exp (-b x)]$, with $a, b>0$. These functions are typical of what might be expected in real biological situations (Wilkinson, 1984; Hart \& Hart., 1992) and are basis functions in the literature of the CPD (Killingback et al., 1999; Killingback \& Doebeli, 2002; Ifti et al., 2004). For these basis functions, one has $x_{\max }=-\log (C /(a b)) / b$, thus, $x_{\max } \geq 1$ if and only if $C<a b \exp (-b)$. In our parametric analysis, we fix $a$ and $b$ and vary $C$ between 0 and $a b \exp (-b)$. One additional remark:

$$
a\left(1-\exp ^{-b x}\right) \rightarrow B x
$$

when $b \rightarrow 0^{+}$and $a \rightarrow+\infty$, with $a b \rightarrow B<\infty$. In fact, for any investment $0<x<1$, the convergence is very fast. Therefore, linear benefit functions, used by Wahl \& Nowak (1999a,b), are an interesting limit case of the basis functions and, because of their simplicity, are especially considered in our analysis.

In the spatial CPD, individuals are placed on the nodes of a network and recollect payoffs from their pairwise interactions with their neighbors 
accordingly with a CPD. The fitness of each individual is given by the sum of these payoffs. At each time step (generation), each individual updates her investment by imitating the investment of her neighbor (including herself) with the highest fitness. The resulting individual (the offspring) responds to the evolutionary principle concerning the clonal reproduction of the phenotype with the highest fitness (Nowak \& May, 1992). Additionally, and this is an important ingredient in the model, Killingback et al. (1999) consider occasional errors at the imitation moment (mutations) that can change the investment of some offspring. It is clear that mutation and limited local interactions are crucial in promoting (even to keep) cooperation in the spatial CPD. In fact, if each individual interacts with the whole population and there is no mutation, each offspring always invests the lowest value from the initial generation. Spatial structures that promote cooperation in the CPD have been previously studied by Killingback et al. (1999); Koella (2000); Ifti et al. (2004). However, as occurs in the standard Prisoner's Dilemma on regular networks (Doebeli \& Hauert, 2005; Hauert, 2006; Jiménez et al., 2008), we argue for the spatial CPD that the spatial structure is capable of sustaining cooperation only in limited cost/benefit regimes. To study this issue, we consider in the spatial CPD on a square lattice with periodic boundary conditions, with individuals interacting with their four nearest neighbors (von Neumann neighborhood) and synchronous updating.

\section{Asymptotics for different cost regimes}

Let us analyze the evolution of the social network defined in Section 2 in the simplest scenario. First, we consider an initial condition with a single individual that invests $x<y$ in a group of $y$-individuals (individuals who invest $y$ ) and assume no mutations. In that case, the update rule is deterministic and we can compute analytically the evolution of the system. More precisely:

(i) If $C(y)-C(x)<(B(y)-B(x)) / 4$, the $x$-individual invades only its four nearest neighbors.

(ii) If $(B(y)-B(x)) / 4<C(y)-C(x)<(B(y)-B(x)) / 2$, the $x$-individual spreads over the lattice, reaching a structure with the same shape of the cross with sawtooth boundaries showed by Jiménez et al. (2008).

(iii) If $C(y)-C(x)>(B(y)-B(x)) / 2$, the $x$-individual spreads until the extinction of the $y$-individuals. 
Assuming that cost and benefit are differentiable functions, and using the fact that they are increasing and concave, it is easy to establish

$$
\frac{C^{\prime}(1)}{B^{\prime}(0)}<\frac{C(y)-C(x)}{B(y)-B(x)}<\frac{C^{\prime}(0)}{B^{\prime}(1)}
$$

for all $0<x<y<1$. Thus, the quotients $C^{\prime}(1) / B^{\prime}(0)$ and $C^{\prime}(0) / B^{\prime}(1)$ provide sufficient and necessary conditions, regardless of the values $x$ and $y$, to determine which absorbing state is reached. For all $0<x<y<1$, from a lattice of $y$-individuals, except one who that invests $x$, the condition $C^{\prime}(0)>B^{\prime}(1) / 4$ is necessary for the spread over all the lattice of the smallest investment and the condition $C^{\prime}(1)>B^{\prime}(0) / 4$ is sufficient. Additionally, $C^{\prime}(1)>B^{\prime}(0) / 2$ is sufficient for the extinction of the highest investment. In our study, we discriminate accordingly the different payoff regimes:

- Low cost, when $C^{\prime}(0)<B^{\prime}(1) / 4$.

- Low-medium cost, if $B^{\prime}(1) / 4<C^{\prime}(0)$ and $C^{\prime}(1)<B^{\prime}(0) / 4$.

- High-medium cost, ifhen $C^{\prime}(1)>B^{\prime}(0) / 4$.

- High cost, if $C^{\prime}(1)>B^{\prime}(0) / 2$.

For simplicity, sometimes we will not distinguish between low-medium and high-medium costs and we refer to both as medium costs. Notice that if the benefit is a linear function, namely $B(x)=B x$, the only low-medium cost is the linear function $C x$, with $C=B / 4$. In that case, $B / 4$ and $B / 2$ are threshold values to determine the region of low, medium and high costs.

\subsection{Asymptotics from random initial conditions without mu- tation}

If a lower-investing individual spreads into a lattice of higher-investing individuals), two lower-investing individuals do the same. Furthermore, for high-medium costs, if there are different low investments into a lattice of higher-investing individuals, at least the lowest spreads over the whole system and other low investments can locally do the same. If the initial investments are randomly chosen, the previous argument, and the fact that investments are initially well-mixed on the network, allow us to conjecture that high-medium cost is sufficient for the spreading of lower investments from random initial conditions. On the other hand, as we argued above, at high costs the smallest investment always dominates the network. In 
contrast, if the cost is low, the small investments do not spread on the system and limited local interactions of the lattice are capable of maintaining cooperation. If the cost is low-medium, we do not provide arguments for determining the asymptotic behavior of the system from a random initial condition. For this reason, it is advisable to first take a look at the linear benefit case, where low-medium costs are reduced only to one threshold value.

The type of social network that we are studying typically reaches a steady state where the mean investment lightly fluctuates around a value, called the asymptotic mean investment. Thus, the promotion or sustaining of cooperation can be contrasted by comparing the asymptotic mean investment with the initial one. Figure 1 shows the typical evolution in time of the mean investment as well as the investment configuration at the steady state for the different cost regimes. For this illustration, we considered the same random initial configuration, uniformly distributed on $[0,1]$ random variables placed on the $100 \times 100$ lattice. For low costs, the figure displays the previously reported clusterization of high investments and consequent promotion of cooperation. However, the figure also illustrates the spread of small investments for medium cost regimes as well as the domination of the lowest for high costs. That is, the square lattice is only capable of promoting (even sustaining) cooperation in low cost regimes. To have an overview of the asymptotic behavior of the investments for different costs we considered different basis functions and varied the cost along its range. For each parametric set, we perform simulations for 100 initial random configurations until the steady state. Although the asymptotic mean investment depends on the initial condition, we noted that its slope, in terms of the cost, is strongly independent. For this reason, we use the average, over the initial random conditions, as a smooth indicator of the relation between costs and the asymptotic mean investment. The slope for four illustrative cases are displayed in Figure 2. We remark that for costs above the threshold cost $B^{\prime}(0) / 2=a b / 2$ the lowest investment dominates the network from any random initial conditions. Note that the transition between significant high asymptotic levels of cooperation to low levels occurs in low-medium costs, namely $B^{\prime}(1) / 4<C<B^{\prime}(0) / 4$. This transition is discontinuous if the benefit is the linear function $B x$. In that case, the phase transition occurs at the threshold cost $B / 4$. The bifurcation of the mean investment time series around this critical cost can be observed in Fig. 1, by comparing the resulting time series for costs $B / 4-\delta$ and $B / 4-\delta$, with small $\delta$. 

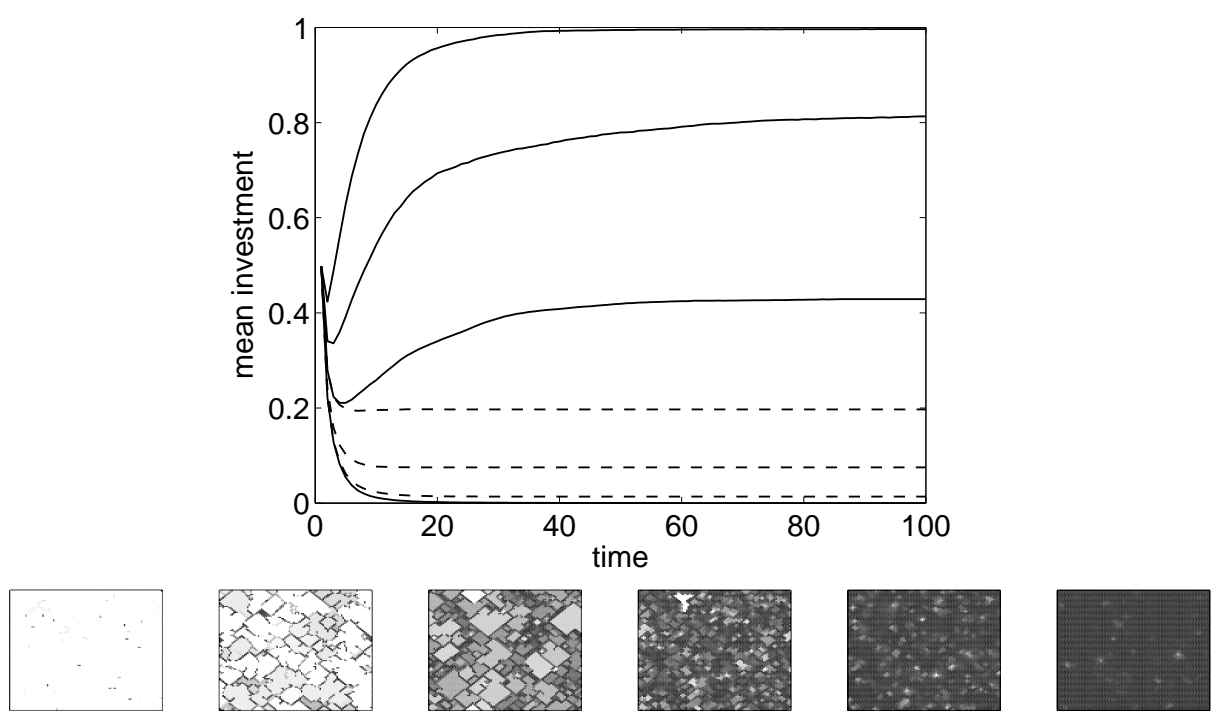

Figure 1: Time series of mean investments and configuration of the lattices at the steady state for linear benefit and low (solid lines), medium (dashed lines) and high (bottom solid line) costs, from the same random initial investments. The figure illustrates the typical behavior close to the threshold costs and in the middle values. The top solid lines and the first three snapshots correspond, respectively, to low costs, $\left\{\delta, \frac{1}{8} B, \frac{1}{4} B-\delta\right\}$, with $\delta=B / 1000$. The dashed lines and the last three snapshots to medium costs $\left\{\frac{1}{4} B+\delta, \frac{3}{8} B, \frac{1}{2} B-\delta\right\}$. The bottom solid line corresponds to high cost $\frac{1}{2} B+\delta$ (its snapshot at the steady state is omitted, a complete black square). The gray scale used to represent the investments on the lattice is linear between black $=0$ and white $=1$. 


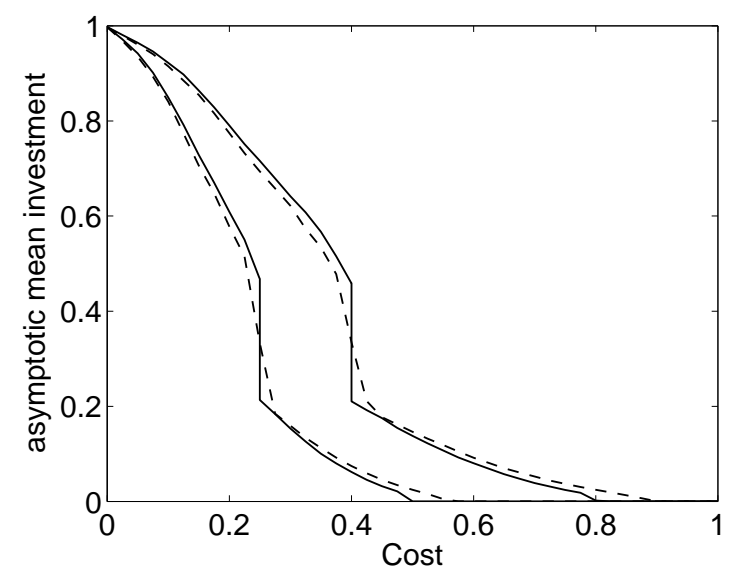

Figure 2: Asymptotic mean investment as function of the cost. The left solid line corresponds to the linear benefit $B(x)=x$ and the right one to $B(x)=8 x / 5$. Left dashed line corresponds to the benefit function $B(x)=$ $5(1-\exp (-2 x / 9))$ and the right one to $B(x)=8(1-\exp (-2 x / 9))$.

\subsection{Asymptotics from small initial investment with mutation}

The mutation mechanism introduced by Killingback et al. (1999) corresponds to neutral variations in the investment pool. Specifically, they consider normal errors, at the imitation moment, with variance equal to $10 \%$ of the expected investment. Without mutation, the mean investment of the network remains into the range of the initial investments. The main result of Killingback et al. (1999) is that starting from arbitrary low investments, occasional mutations can increase the mean investment to significant levels, after a long runtime. Their simulations are based on a parametric set that we have characterized as low cost. Namely, $B(x)=8(1-\exp (-x))$, $C(x)=0.7 x$, and $x_{\max } \approx 2.4361$. Performing simulations with the same mutation rate per individual (1/100) and modeling errors in the same way, we confirm their main result for any low cost, when the benefit function is not linear. Moreover, we are now prepared to explain why mutation raises cooperation for this cost/benefit regime: When costs are low, our results show that negative mutations (i.e. mutations with lower investments than their expectations) do not spread on the system and that higher-investing clusters invade lower-investment ones. To see this, notice that individuals in the boundaries of the higher-investing cluster outperform the individu- 
als in the boundaries of the lower-investing cluster. On the other hand, interactions with eventual positive mutations (mutations with investments higher than their expectations) can give origin to a small cluster of higher investments, again in the low cost regime. For a strictly concave benefit function, the lower the investment the higher the additional payoff that a positive mutant adds to their neighbors. Additionally, the more concave the benefit function is, the stronger such effect is. To sum up, in this unbeatable cost/benefit framework (e.g. low cost and very concave benefit function), a positive mutant can gradually invade a world of low investors until obtaining a high mean investment at the steady state. However, the less concave the benefit function is the lower the mean investment reached is. This pattern coincides with the results of Le Gaillard et al. (2003), who analyze different regimes of physiological costs in a related adaptive dynamics with the CPD. Our results complement the analysis for lattice-structured population. In fact:

1. If the benefit is linear, the lattice-structured population is only capable of sustaining the mean investment around the initial one, even if the cost is low.

When significant costs are considered, other asymptotic results occur:

2. If the cost is low-medium, for any basis benefit function (linear or strictly concave), the lattice is only capable of sustaining the mean investment around the initial one. This occurs with and without mutation. A mutation (negative or positive) does not have any effect on the lattice.

3. In high-medium cost regimes, the mutation mechanism works against cooperation, by favoring negative mutations (i.e. mutations with lower investments than the expectations). Thus, if there are not mutations, the lattice can sustain low levels of cooperation, but cannot stop the fall of investments if any occasional negative mutation appears on the lattice.

4. For high costs, the investments nose dive, with or without mutation.

Summarizing, small mutations promote cooperation only in very particular scenarios of low costs of cooperation. 


\section{Effect of Crossover in the evolution of coopera- tion}

The above analysis was obtained when the strategy update rule is based on clonation. Now we consider arithmetic crossover (Eiben \& Smith, 2007) as the way to update investments. Instead of focal individuals copying the best neighbor, each individuals updates her investment with a new one "between" her own investment and the investment of her successful neighbor. Into the spatial CPD, arithmetic crossover operates as follows: If an individual invests $x$ and her successful neighbor invests $y$, in the next generation, the former will invest

$$
\alpha x+(1-\alpha) y
$$

plus a possible error, for some $\alpha \in[0,1]$. In an economic and social scenario, the parameter $\alpha$ can be viewed as a factor of resistance to learning or as a measure of wariness. When $\alpha=0$, there is no resistance, individuals copy the strategy of their neighbors with the highest payoff. However, when $0<\alpha<1$ the individuals gradually learn from their most successful partner. Whereas if $\alpha=1$ individuals refuse to learn.

\subsection{Supporting cooperation in adverse cost regimes}

As in Section 3, we analyze first the evolution in time of the social network without errors in the update rule. Similar to the previously studied case, the lattice reaches a steady state where the mean investment lightly fluctuates around a constant. Figure 3 shows the typical evolution in time of the mean investment for different resistance levels in medium and high costs. It is noteworthy how the presence of resistance to learning can increase the asymptotic mean investment to higher levels when investments are costly. To have a better understanding of the effect of resistance to learning, we considered different cost/benefit functions and varied the resistance along its range. For each parametric set, we performed simulations for 100 initial random configurations until the steady state was reached. We observe that only small variations on the asymptotic mean investments were observed. Thus, the average, over the initial random conditions, is a robust estimator of this asymptotic mean investment, regardless the initial condition. In general, in low cost regimes, resistance neither favors cooperation nor dulls it. However, for any medium or high cost, resistance to learning strongly favors cooperation. Moreover, the higher the resistance to learning is the larger the mean investment asymptotically reached. Figure 4 characterizes the 


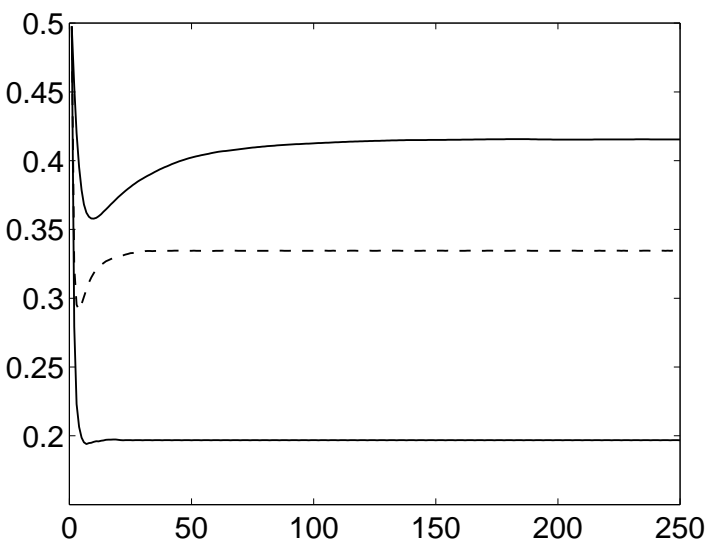

(a) medium cost

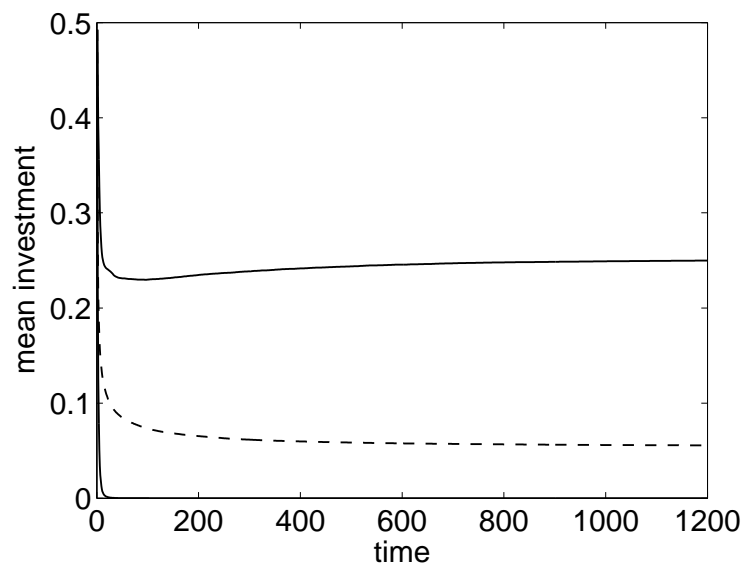

(b) high cost

Figure 3: Time series of mean investments for medium (a) and high (b) cost with different resistances to learning. Top solid lines correspond to $\alpha=0.8$, dashed lines to $\alpha=0.2$ and bottom solid line to $\alpha=0$. The benefit function is linear, and the costs considered are to the right of the thresholds $\frac{1}{4} B$ and $\frac{1}{2} B$. 
behavior for the different cost regimes. We conclude that cautious learning is more efficient than hasty learning when the cost is medium or high and it is necessary to sustain cooperation (even in low levels) for high costs.

To understand how resistance works to favor cooperation in medium/high cost regimes, let us consider again the simplest initial condition with a single $x$-individual in a lattice occupied with $y$-individuals, with $x<y$. Notice that the $x$-investment spreads on the lattice (or dominates it) if individuals have no resistance to learning. For this initial configuration, let the epicenter be the individual with initial investment $x$ and the front of the wave be the $y$-individuals closer to the epicenter. It is easy to show that while individuals gradually learn from the individual with the least investment (expansion of the wave), the investment at the front of the wave of the generation $k$ changes to $y-(1-\alpha)^{k}(y-x)$ in the next generation. Thus, if the lattice is big enough, there exists a resistance value such that these individuals at the front of wave learn from their neighbors with higher investments, in some generation, producing a reflection of the wave. An immediate consequence of this result is that the expansive wave generated by the $x$-agent is reflected by a barrier of $y$-individuals when those have enough resistance to learning. The reflected wave is propagated towards the epicenter making an increment of the mean investment of the system. Thus, if the lattice is big enough and individuals have enough resistance to learning, the system can asymptotically recover high investments (defection absorption); even if there is not enough resistance to learning, the wave can be attenuated producing a diminishment in the mean investment (defection attenuation). Moreover, the absorption/attenuation effect, illustrated in Figures 5 and 6, can locally operate for random initial configuration until producing the rebound in the mean investment evolution showed in Figure 3(a).

\subsection{The origin of cooperation: Learning errors and resis- tance to learning}

The most amazing results occur when there are occasional errors in the gradual learning rule. To study this phenomenon, we performed simulations by covering low, medium and high costs and linear and strictly concave benefit functions. As in Section 3.2, we considered low initial investments, uniformly distributed between zero and $1 / 10$, and error rate of $1 \%$, Gaussian distributed with variance equal to $10 \%$ of the expected investment. Our study is conclusive as to the effect of the resistance to learning:

1. In medium costs (low-medium and high-medium) as well as in low costs with linear benefits, where the model with learning errors and 


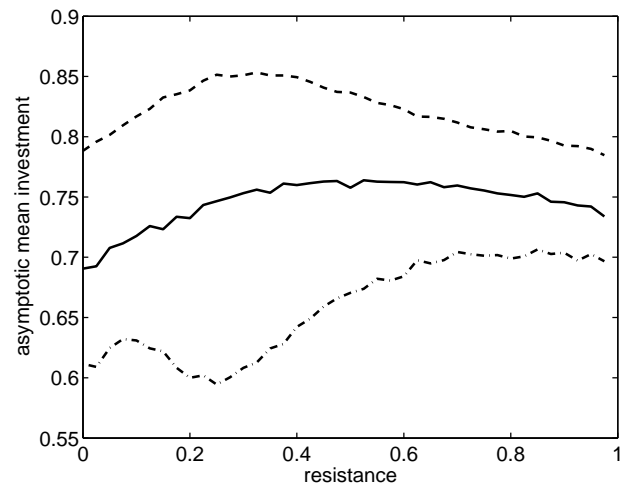

(a) low cost

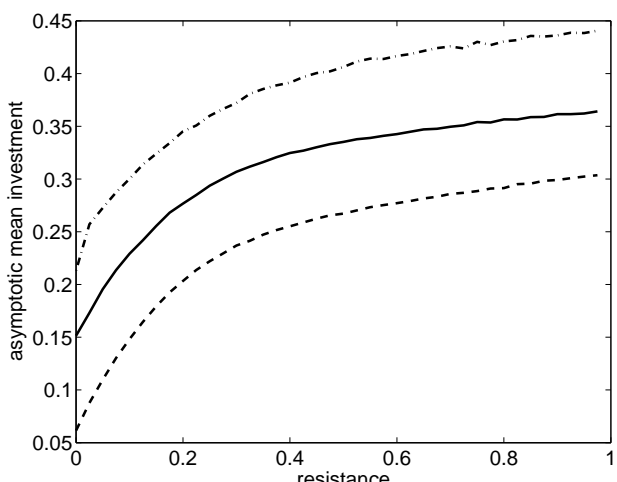

(b) med cost

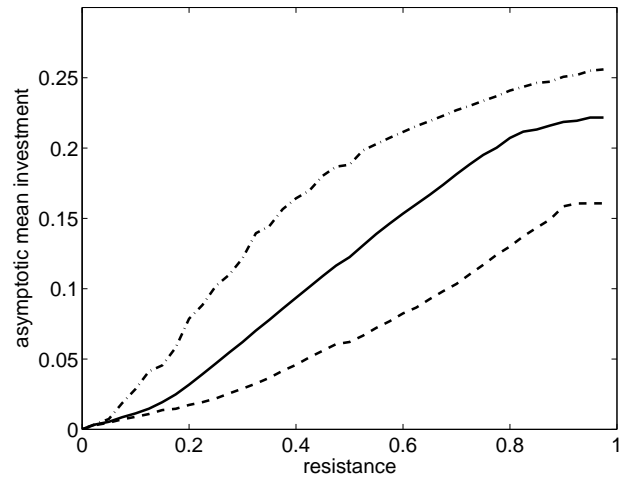

(c) high cost

Figure 4: Asymptotic mean investment as a function of $\alpha$ for the benefit function $B x$. Low costs in (a): top dashed line $\frac{1}{8} B$, solid line $\frac{1}{6} B$, and bottom dashed line $\frac{1}{5} B$. Medium costs in (b): top dashed line $\frac{1}{4} B+\delta$, solid line $\frac{3}{10} B$, and bottom dashed line $\frac{2}{5} B$. High costs in (c): top dashed line $\frac{1}{2} B+\delta$, solid line $\frac{3}{5} B$, and bottom dashed line $\frac{3}{4} B$. To consider costs close to the thresholds we make $\delta=1 / 1000$. 

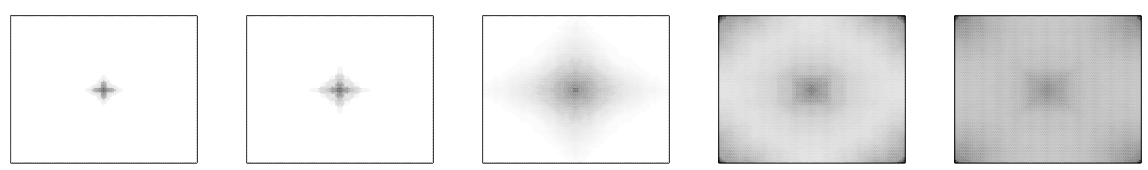

Figure 5: Attenuation of a 0 -investor for a pool of 1-investors with low resistance to learning at time generations 10, 20, 100, 300, 1000 and 3000. $B(x)=4(1-\exp (-x / 2)), C=0.8$, and $\alpha=0.25$. The asymptotic mean investment in the stationary state is around 0.67. Note: for this parametric set, the 0 -invertor dominates the system if there is no resistance to learning.
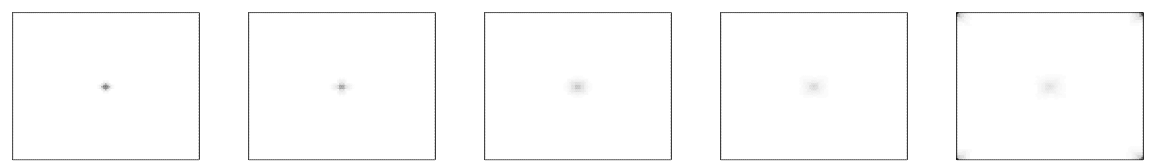

Figure 6: Absorption of a 0-investor for a pool of 1-investors with enough resistance to learning at time generations 10, 20, 100, 300, 1000 and 3000. $B(x)=4(1-\exp (-x / 2)), C=0.8, \alpha=0.65$. The asymptotic mean investment in the stationary state is almost 1.

without resistance is only capable of sustaining cooperation in the initial investment range, gradual learning leads to significant higher asymptotic mean investment.

2. In high costs, occasional errors work against cooperation and even the resistance to learning is unable to maintain cooperation.

Figure 7 shows the typical effect of the resistance to learning in adverse conditions in which simple mutations in the structured population are not sufficient to trigger high levels of cooperation. In these scenarios of low and medium costs, with linear or strictly concave benefits, there always exists a value for the resistance parameter to raise cooperation to higher levels. Moreover, we observed through simulations, that any resistance level $(0<\alpha<1))$ is enough to promote cooperation when costs are low. Medium levels of resistance may be required when costs are medium; the closer the cost is to the high cost threshold, the higher the level of resistance required.

\section{Conclusions}

In this paper we have studied the effect in the evolution of cooperation of introducing arithmetic crossover, instead of clonation, in the strategy up- 


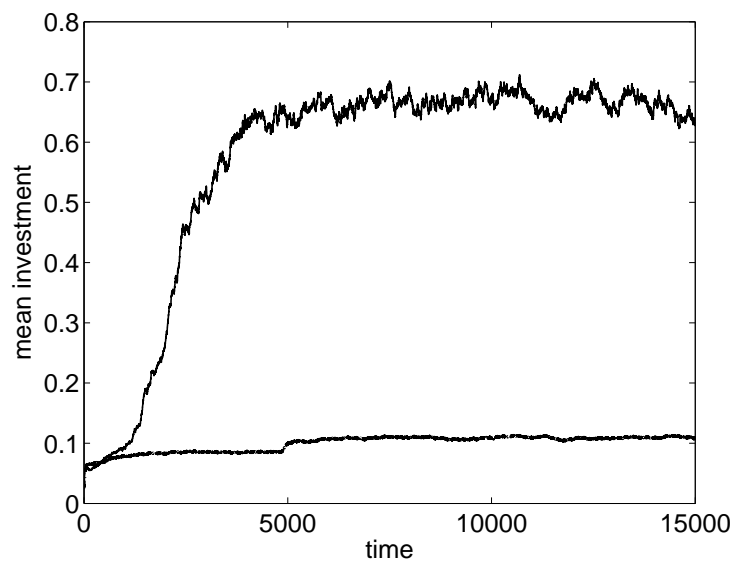

(a)

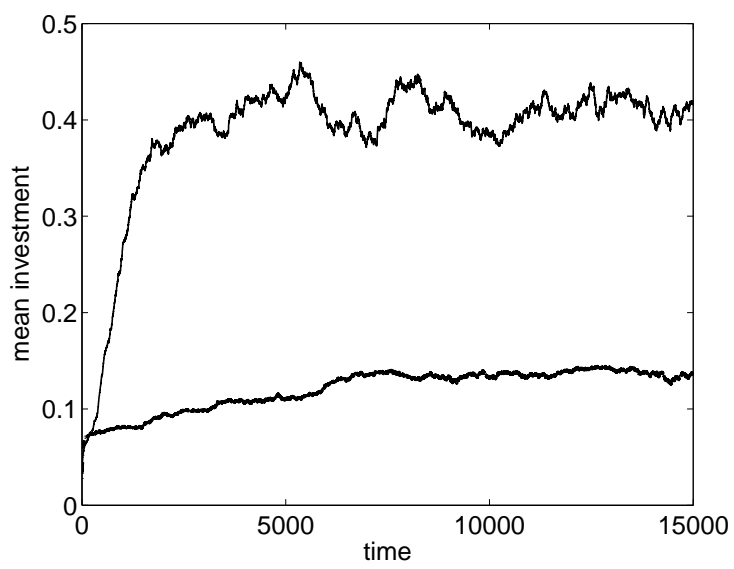

(b)

Figure 7: Time series of mean investments with resistance (top lines) and without resistance (bottom lines): (a) linear benefit $(B=1)$, low cost $(C=$ $0.2)$ and low resistance $(\alpha=0.1)$. (b) strictly concave benefit $(B(x)=$ $4[1-\exp (-x / 2)])$, medium cost $(C=0.4)$ and medium resistance $(\alpha=0.5)$. Gaussian mutation, with variance equal to $10 \%$ of the expected investment and mutation rate $1 \%$. Initial investments are uniformly distributed on $\left[0, \frac{1}{10}\right]$. 
date rule of the spatial CPD. We have reviewed this evolutionary game in detail when the strategy update rule is based on clonation. First, we have studied the model without errors in the copying process. For this case, we have found a simple classification of the payoff regimes (low, low-medium, high-medium, and high costs) which allows us to determine the asymptotic mean behavior of a population structured on a square lattice. From random initial conditions, the spatial structure promotes high investments only in low cost regimes. We observed fast transitions to low mean investments in low-medium costs. It is interesting to notice that these transitions are discontinuous when the payoffs are linear functions. In regimes of high-medium cost the spatial structure is unable to keep the initial mean cooperation investment. The lowest investment in the initial state dominates the system for any high-cost regime. When occasional errors in the copying process are considered, cooperation emerges from a non-cooperative initial state only under a particular regime of low cost and strictly concave benefit. To observe this emergence, previously reported in the literature of the spatial CPD (Killingback et al., 1999; Koella, 2000; Ifti et al., 2004), negligible costs of cooperation (Le Gaillard et al., 2003) are required. For the rest of the regimes, the essential problem of cooperation remains: Occasional errors have no effect on the evolution of cooperation when costs are low and the benefit function is linear or when costs are low-medium. Moreover, they work against cooperation in high-medium and high costs.

Arithmetic crossover can be viewed as a step-by-step learning mechanism in the strategy update process that can prevent individuals from their costly acts. Amazingly, it is this aspect that offers important insight into the problem of origin and sustainment of cooperation. Our analysis explains how cooperation evolves and is maintained when gradual learning works in the spatial CPD. We have proved that the resistance to learning has a significant effect on medium (low-medium and high-medium) cost and high cost regimes: The less that is learnt in a single step, the greater the degree of global cooperation finally attained. When learning errors can occur on an arbitrarily non-cooperative population, we have showed that a higher-investment mutant spreads all over the system raising cooperation to significant levels in regimes of low costs (with linear or non-linear benefits) and medium costs. The larger the cost, the greater the resistance to learning needed in a single step. However, in more adverse conditions of high costs, occasional errors work against cooperation and even the resistance is unable to maintain cooperation.

We call attention to the fact that gradual learning can be introduced in a straightforward way into the strategy update rule of any continuous 
evolutionary game, such as the continuous Snowdrift game (Doebeli et al., 2004) and the spatial ultimatum game (Page et al., 2000). Gradual learning can also operate jointly with different selection mechanisms to identify from whom to learn. To compare results with previous literature on spatial the CPD, we have considered learning from the successful neighbor, but the crossover operator can be easily fitted to random selection criterions (Szabó \& Fáth, 2007). We have only considered arithmetic crossover, because it is the natural rule when phenotypes are represented by real numbers, such as cooperative investments. But different schemas of crossover can be considered when strategies are not scalar. Our formulation is quite simple and can be applied in a wide range of scenarios. We hope that this work will contribute to highlighting the relevant role of this evolutionary principle for the study of cooperation.

In conclusion, resistance to learning provides a natural solution to the evolutionary riddle of the origin and maintenance of cooperation in a world governed by selective forces. It is a fast mechanism for generating diversity, allowing adaptation to transitory changes. Our results suggest that populations which learn slowly from successful partners are, in the long term, more efficient in the sense that they allow for higher cooperative investments. Thus, our model provides an evolutionary version of the tortoise and the hare fable when the goal is cooperation.

Acknowledgements: We gratefully thank Víctor Eguíluz of IFISC for multiple inspiring discussions related to this paper.

\section{References}

Axelrod, R., Hamilton, W. D., 1981. The evolution of cooperation. Science 211, 1390-1396.

Doebeli, M., Hauert, C., 2005. Models of cooperation based on the Prisoner's Dilemma and the Snowdrift game. Ecol. Lett. 8, 748-766.

Doebeli, M., Hauert, C., Killingback, T., 2004. The evolutionary origin of cooperators and defectors. Science 303, 859-862.

Doebeli, M., Knowlton, N., 1998. The evolution of interspecific mutualisms. Proc. Natl Acad. Sci. USA 95, 8676-8680.

Dugatkin, L. A., 1997. Cooperation among animals. Oxford University Press. 
Eiben, A. E., Smith, J. E., 2007. Introduction to Evolutionary Computing, Springer.

Frean, M., 1996. The evolution of degrees of coperation. J. Theor. Biol. 182, 549-559.

Hart, B., Hart, L., 1992. Reciprocal allogrooming in impala. Anim. Behav. 44, 10731083.

Hauert, C., 2006. Spatial effects in social dilemmas. J. Theor. Biol. 240, $627-636$.

Ifti, M., Killingback, T., Doebeli, M., 2004. Effects of neighborhood size and connectivity on the spatial Prisoner's Dilemma. J. Theor. Biol. 231, 97-106.

Jiménez, R., Lugo, H., Cuesta, J., Sánchez, A., 2008. Emergence and resilience of cooperation in the spatial Prisoner's Dilemma via a reward mechanism. J. Theor. Biol. 250, 475-483.

Killingback, T., Doebeli, M., Knowlton, N.,1999. Variable investment, the Continuous Prisioner's Dilemma, and the origin of cooperation. Proc. $R$. Soc. Lond. B 266, 1723-1728.

Killingback, T., Doebeli, M., 2002. The Continuous Prisoner's Dilemma and the evolution of cooperation through reciprocal altruism with variable investment. Am. Nat. 160, 421-438.

Koella, J. C., 2000. The spatial spread of altruism versus the evolutionary response of egoist. Proc. R. Soc. Lond. B 267, 1975-1985.

Le Gaillard, J. F., Ferriere, R., Dieckmann, U., 2003. The adaptive dynamics of altruism in spatially heterogeneous populations. Evolution 57, 1-17.

Lieberman, E., Hauert, C., Nowak, M. A., 2005. Evolutionary dynamics on graphs. Nature 433, 312-316.

Mar, G., St Denis, P., 1994. Chaos in cooperation: continuous-valued Prisoner's Dilemmas in infinite-valued logic. Int. J. Bifur. Chaos 4, 943-958.'

Maynard Smith, J. \& Szathmáry, E., 1995. The Major Transitions in Evolution. Oxford: W. H. Freeman \& Co.

Nowak, M. A., 2006. Five rules for the evolution of cooperation. Science 314, 1560-1563 
Nowak, M. A., May, R. M., 1992. Evolutionary games and spatial chaos. Nature 415, 424-426.

Page, K. M., Nowak, M. A., Sigmund, K., 2000. The spatial ultimatum game. Proc. R. Soc. Lond. B 267, 2177-2182.

Pennisi, E., 2005. How did cooperative behavior evolve? Science 309, 93.

Roberts, G., Sherratt, T. N., 1998. Development of cooperative relationships through increased investment. Nature 394, 175-179.

Sherratt, T. N., Roberts, G., 1999. The Evolution of Quantitatively Responsive Cooperative Trade. J. Theor. Biol. 200, 419-426.

Sherratt, T. N., Roberts, G., 2002. The stability of Cooperation Involving Variable Investment. J. Theor. Biol. 215, 47-56.

Szabó, G., Fáth, G., 2007. Evolutionary games on graphs. Phys. Rep., 446, $97-216$.

Trivers, R., 1971. The evolution of reciprocal altruism. The Q. Rev. Biol., 46, 35-57.

Verhoeff, T., 1993. A Continuous Version of the Prisoners Dilemma. Computing Science Note 93/02. Eindhoven: Eindhoven University of Technology (also available at http:www.win.tue..nl/wstomv/publications/).

Wahl, L. M., Nowak, M. A., 1999. The continuous prisoner's dilemma: I. Linear reactive strategies. J. Theor. Biol. 200, 307-322.

Wahl, L. M., Nowak, M. A., 1999. The continuous prisoner's dilemma: II. Linear reactive strategies with noise. J. Theor. Biol. 200, 323-338.

Wilkinson G. S., 1984. Reciprocal food-sharing in the vampire bat. Nature 308, 181-184. 